\title{
Comparative Effectiveness of Carboplatin/Pemetrexed With Versus Without Bevacizumab for Advanced Nonsquamous Non-Small Cell Lung Cancer
}

Stephen J. Bagley, MD, MSCEa,b; Suzanna Talento, BAc; Nandita Mitra, PhD'; Neal J. Meropol, MDe,f; Roger B. Cohen, MDa,b; Corey J. Langer, MD ${ }^{a, b}$; and Anil Vachani, MD, MSCEa,g,h

\section{ABSTRACT}

Background: Despite recent advances in targeted therapy and immunotherapy for advanced non-small cell lung cancer (NSCLC), carboplatin/pemetrexed/bevacizumab remains a commonly used first-line regimen. However, it is unknown whether the addition of bevacizumab to carboplatin/pemetrexed improves overall survival (OS). Materials and Methods: Using nationally representative curated electronic health record data from Flatiron Health, we performed a retrospective cohort study of patients diagnosed with advanced nonsquamous NSCLC who received $\geq 1$ cycle of carboplatin/pemetrexed \pm bevacizumab as initial systemic therapy for stage IV or metastatic/recurrent disease. The OS impact of adding bevacizumab to carboplatin/pemetrexed was assessed using a Cox proportional hazards model to adjust for age, sex, race, original tumor stage, time between diagnosis of metastatic disease and start of chemotherapy, and performance status. In a secondary analysis of patients at a single academic institution, we also adjusted for the presence of brain metastases, hemoptysis, and anticoagulation. Results: A total of 4,724 patients were included, of which 2,759 patients (58\%) received carboplatin/pemetrexed and 1,965 (42\%) received carboplatin/pemetrexed/bevacizumab. Median OS was 12.1 months ( $95 \% \mathrm{Cl}, 11.2-12.9$ months) in the carboplatin/pemetrexed/ bevacizumab group compared with 8.6 months ( $95 \% \mathrm{Cl}$, 8.1-9.1 months) in the carboplatin/pemetrexed group $(P<.001)$. Bevacizumab use remained associated with improved $O S$ in a multivariate model (hazard ratio, $0.80 ; 95 \% \mathrm{Cl}, 0.75-0.86 ; P<.001$ ). In the secondary, institutional analysis $(\mathrm{N}=539)$, the effect of bevacizumab was unchanged (hazard ratio, $0.75 ; 95 \% \mathrm{Cl}, 0.59-0.96 ; P=.02$ ). Conclusions: In this large, real-world dataset, the addition of bevacizumab to firstline carboplatin/pemetrexed for metastatic nonsquamous NSCLC was associated with improved OS.

J Natl Compr Canc Netw 2019;17(5):469-477 doi: 10.6004/jnccn.2018.7102

\footnotetext{
${ }^{a}$ Division of Hematology/Oncology, Perelman School of Medicine at the University of Pennsylvania, and ' $\mathrm{Abramson}$ Cancer Center, Philadelphia, Pennsylvania; 'Tufts University School of Medicine, Boston, Massachusetts; ${ }^{d}$ Department of Biostatistics, Epidemiology, and Informatics, Perelman School of Medicine at the University of Pennsylvania, Philadelphia, Pennsylvania; ${ }^{\mathrm{e}}$ Flatiron Health, New York, New York; ${ }^{f}$ Case Comprehensive Cancer Center, Case Western Reserve University, Cleveland, Ohio; and PDivision of Pulmonary, Allergy, and Critical Care, Perelman School of Medicine at the University of Pennsylvania, and hepartment of Medicine, Corporal Michael J. Crescenz VA Medical Center, Philadelphia, Pennsylvania.
}

\section{Background}

For patients with advanced non-small cell lung cancer (NSCLC) whose tumors do not harbor an actionable genomic alteration or have PD-L1 expression (tumor proportion score $\geq 50 \%$ ), a platinum-based chemotherapy doublet remains a standard component of first-line therapy. ${ }^{1,2}$ Multiple cytotoxic agents are recommended as partners for platinum based on clinical trial data and expert opinion, ${ }^{3}$ and the choice is often based on histology. ${ }^{3}$ Use of pemetrexed for nonsquamous tumors is supported by subgroup analysis of a 2008 randomized trial, which demonstrated superior overall survival (OS) in patients with nonsquamous histology who received cisplatin/pemetrexed compared with cisplatin/ gemcitabine. ${ }^{4}$ Despite debate regarding interpretation of this trial, ${ }^{5}$ carboplatin/pemetrexed is the most commonly used first-line chemotherapy regimen in the United States for advanced nonsquamous NSCLC. ${ }^{6,7}$

Although recent data have established that the addition of the immune checkpoint inhibitor pembrolizumab to first-line carboplatin/pemetrexed improves OS in advanced nonsquamous NSCLC regardless of PD-L1 expression, ${ }^{8}$ some patients are unable to receive PD-1 inhibitors because of preexisting autoimmune disease ${ }^{9}$ or lack of access. ${ }^{10}$ When these patients lack a targetable mutation, carboplatin/pemetrexed alone or with the antiangiogenic monoclonal antibody bevacizumab remains a relevant systemic regimen. Bevacizumab was initially approved for nonsquamous NSCLC in 2006 based on results of the randomized phase III ECOG 4599 trial, which demonstrated an OS advantage associated with addition of bevacizumab to carboplatin/ paclitaxel. ${ }^{11}$ Although results of the PointBreak trial showed that carboplatin/pemetrexed/bevacizumab yielded similar OS compared with carboplatin/paclitaxel/bevacizumab, but with less toxicity, ${ }^{12}$ there has never been a randomized controlled trial (RCT) to demonstrate that OS is improved by adding bevacizumab to carboplatin/ pemetrexed. Thus, despite frequent use of this regimen 
in clinical practice, current ASCO clinical practice guidelines state that there is insufficient evidence to recommend bevacizumab in combination with pemetrexed/carboplatin. ${ }^{2}$ Because of this significant gap in the literature, we used nationally representative electronic health record (EHR) data from Flatiron Health to compare the effectiveness of first-line carboplatin/ pemetrexed \pm bevacizumab in patients with metastatic nonsquamous NSCLC. We also supplemented these data with our institutional experience to account for confounding clinical variables not captured in the Flatiron database.

\section{Materials and Methods}

\section{Data Source}

We conducted a retrospective cohort study using deidentified EHR data from the Flatiron Health database. ${ }^{13}$ Information in this database comprises structured data (eg, cancer-related diagnoses/staging, laboratory data, medications) and abstracted data from unstructured documents in EHRs (eg, physician notes, radiology/ pathology/biomarker reports, discharge summaries). The database provides longitudinal, patient-level data, including demographics, treatments, recurrence patterns, and survival data, and is generalizable to the national population in terms of age, sex, and geography. ${ }^{14}$ The dataset delivered for this study had a cutoff of June 30, 2017 (inclusive), and represented $>260$ community cancer clinics, ranging from small practices to large multicenter practices. Both Central and University of Pennsylvania Institutional Review Board approvals were obtained.

\section{Study Population}

The main study cohort included patients with histopathologically or cytologically proven nonsquamous NSCLC with a confirmed diagnosis of de novo stage IV disease or recurrence of earlier stage disease on or after January 1, 2011, and who had started first-line chemotherapy with carboplatin/pemetrexed \pm bevacizumab within 4 months of diagnosis of metastatic/recurrent disease. At least 6 months of follow-up between chemotherapy initiation and end of the observation period (June 30, 2017) was required. To identify first-line chemotherapy regimens, each patient was assigned an index date, defined as the date of diagnosis with metastatic/ recurrent NSCLC. Start of first-line systemic therapy was defined as the first episode of an eligible therapy (any component of carboplatin/pemetrexed \pm bevacizumab) given after or up to 14 days before the index date. To be considered as having received one of these regimens, the start date for each drug in the regimen had to have been within 28 days of the start dates for the other drugs.
Patients with initial stage I-III NSCLC were allowed if carboplatin/pemetrexed \pm bevacizumab was administered as the first treatment for recurrence. Patients must have had at least 2 documented clinical visits in the database on or after January 1, 2011. Patients with EGFR or $A L K$ rearrangements and/or who were treated with anti-PD-1/PD-L1 or targeted oral agents as first-line therapy were excluded. Finally, patients treated at academic centers, which represented $6.2 \%$ of the full Flatiron cohort, were excluded due to incomplete structured data for these patients in the database before 2014. The national cohort assembly strategy is detailed in Figure 1.

In addition to the national cohort, we performed a separate analysis of patients treated at the University of Pennsylvania who met the same inclusion criteria. These patients were present in the Flatiron database but were excluded from the dataset used for the national cohort analysis. This single site analysis allowed for manual chart abstraction for key variables that could have confounded the relationship between bevacizumab and OS and were not available in the national cohort, including brain metastases, hemoptysis, or anticoagulation use.

\section{Survival Outcomes}

The primary outcome was OS, defined as the number of months from administration of first chemotherapy agent for metastatic disease until the date of death or end of the observation period (June 30, 2017). Patients not deceased by the end of the observation period were censored at date of last follow-up. Flatiron staff curates mortality information from structured fields and unstructured documents within the EHR, the Social Security Death Index, and a commercial death dataset that mines data from obituaries and funeral homes. Flatiron's mortality data are validated through direct comparisons with the National Death Index and by comparing the results of analyses using Flatiron mortality data with results from published literature and the SEER database. Flatiron's mortality data are estimated to have a sensitivity of $85 \%$ to $90 \%$, specificity of $97 \%$ to $98 \%$, and accuracy of $95 \%$ to $97 \% .^{15}$

\section{Baseline Characteristics}

Demographic and clinical data obtained for this study included age (categorized as <55, 55-64, 65-74, and $\geq 75$ years), sex, race (white, black, Asian, other, and unknown), initial stage of NSCLC, number of days between diagnosis of metastatic NSCLC and start of first-line chemotherapy, and ECOG performance status (PS) within 60 days of first administration of chemotherapy (most proximate value to first dose of chemotherapy was recorded). ECOG PS was categorized as 0, 1, 2, 3 to 4, or 


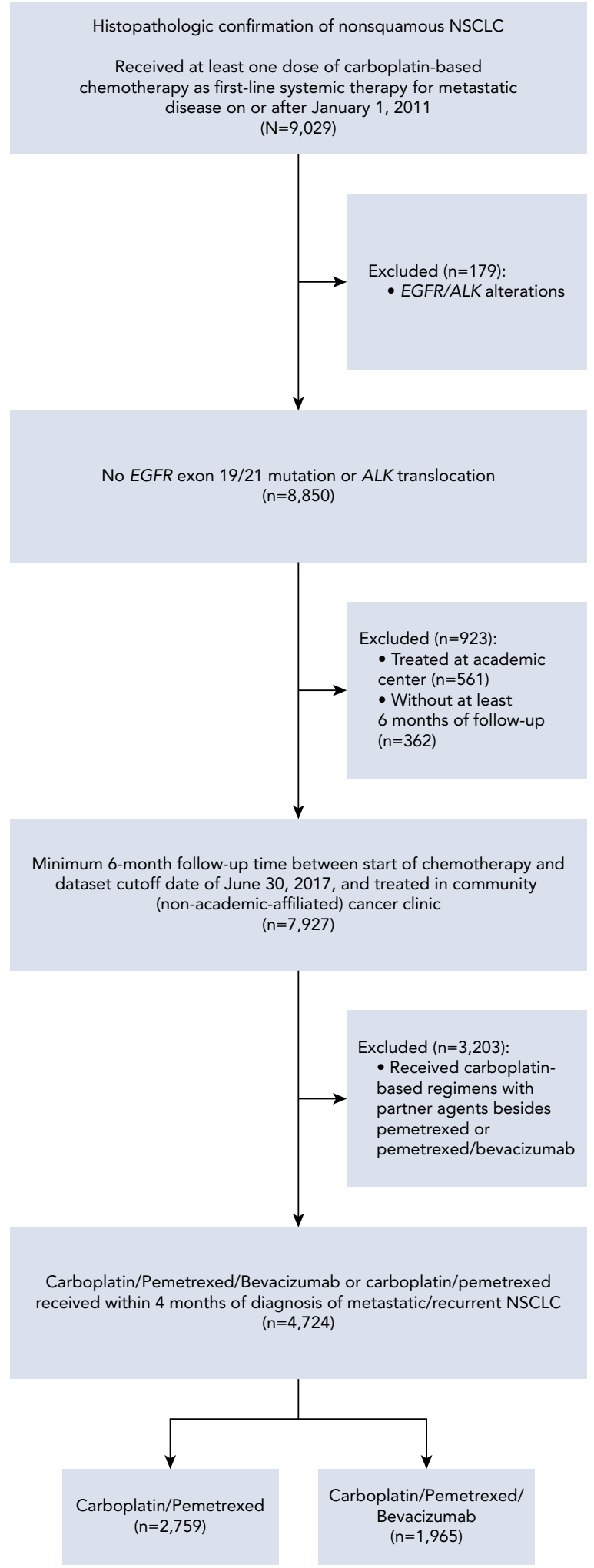

Figure 1. National cohort definition.

Abbreviation: NSCLC, non-small cell lung cancer. missing (ie, no ECOG PS recorded within 60 days of chemotherapy start).

In the University of Pennsylvania cohort, manual chart abstraction from the EHR was performed to collect data on brain metastases (present or absent), hemoptysis (present or absent at any time since diagnosis of metastatic NSCLC), and use of therapeutic anticoagulation (low-molecular-weight heparin, warfarin, or a direct oral anticoagulant; present or absent) at the time of chemotherapy initiation. These variables were chosen as potential confounders because of their importance in physician decision-making with regard to bevacizumab use and their potential impact on survival.

\section{Statistical Analysis}

Categorical variables were reported as frequency and percentage, and continuous variables were reported as median, interquartile range (IQR), mean, and standard deviation. We compared baseline characteristics between treatment groups using chi-square tests for categorical variables and 2-sample $t$ tests for continuous variables. The Kaplan-Meier method was used to estimate median OS, and a log-rank test was used to test for crude differences between the groups. Median follow-up was calculated using the reverse Kaplan-Meier method. Cox proportional hazards regression was used to estimate a hazard ratio (HR) adjusted for all demographic and clinical characteristics in Table 1 (time between diagnosis of metastatic disease and chemotherapy initiation was categorized as quartiles). The inclusion of an interaction term for treatment regimen and age was evaluated. Proportional hazards assumption was evaluated using graphic methods (complementary log-log plot) and did not indicate deviations from proportionality. We also performed sensitivity analyses restricted to patients with known ECOG PS and with extreme case imputation for ECOG PS (all patients with unknown PS who received bevacizumab were assumed to have ECOG PS 1; those with unknown PS who did not receive bevacizumab were assumed to have ECOG PS 2), and subgroup analyses were restricted to elderly patients. In addition, a secondary analysis was performed applying the same Cox proportional hazards model described earlier to the University of Pennsylvania cohort. In this institutional cohort, the model was expanded to include brain metastases, hemoptysis, and therapeutic anticoagulation.

$P<.05$ was considered statistically significant. All tests were 2-tailed. STATA 14 was used for statistical analyses (StataCorp LLP).

\section{Results}

\section{Patient Demographics}

In the national cohort, we identified 4,724 patients diagnosed with metastatic nonsquamous NSCLC who 
Table 1. Baseline Patient Characteristics in the National Cohort

\begin{tabular}{|c|c|c|c|}
\hline Characteristic & $\begin{array}{l}\text { Carboplatin/Pemetrexed } \\
\qquad(\mathrm{N}=\mathbf{2 , 7 5 9 )}\end{array}$ & $\begin{array}{l}\text { Carboplatin/Pemetrexed/Bevacizumab } \\
\qquad(\mathrm{N}=1,965)\end{array}$ & $P$ Value \\
\hline Age, y & & & $<.001$ \\
\hline $55-64$ & $721(26.1 \%)$ & $580(29.5 \%)$ & \\
\hline $65-74$ & $1,024(37.1 \%)$ & $774(39.4 \%)$ & \\
\hline Female & $1,320(47.8 \%)$ & $977(49.7 \%)$ & \\
\hline Male & $1,439(52.2 \%)$ & $988(50.3 \%)$ & \\
\hline Race & & & .34 \\
\hline White & $1,832(66.5 \%)$ & $1,313(66.8 \%)$ & \\
\hline Unknown & 456 (16.5\%) & 309 (15.8\%) & \\
\hline Stage & & & .02 \\
\hline Metastatic recurrence of stage I-III & $475(17.2 \%)$ & $288(14.7 \%)$ & \\
\hline Initial stage IV & $2,284(82.8 \%)$ & $1,677(85.3 \%)$ & \\
\hline \multicolumn{2}{|c|}{ Median days from metastatic diagnosis to chemotherapy initiation } & & .01 \\
\hline Quartile 1 (0-21) & 715 (25.9\%) & $537(27.3 \%)$ & \\
\hline Quartile 2 (22-33) & $685(24.8 \%)$ & $538(27.4 \%)$ & \\
\hline Quartile 3 (34-50) & 715 (25.9\%) & $492(25.0 \%)$ & \\
\hline Quartile 4 (51-120) & $644(23.4 \%)$ & $398(20.3 \%)$ & \\
\hline
\end{tabular}

started first-line carboplatin/pemetrexed \pm bevacizumab between January 1, 2011, and January 30, 2017. Median time between diagnosis of metastatic disease and chemotherapy initiation was 32 days (IQR, 21-48 days). Patients receiving bevacizumab were more likely to have ECOG PS 0 (22.1\% vs $17.8 \% ; P<.001)$ and less likely to have ECOG PS 2 (6.9\% vs $9.9 \% ; P<.001)$ compared with those not receiving bevacizumab. In addition, those receiving bevacizumab were more likely to have been treated for stage IV disease (de novo metastatic) than for recurrence ( $85.3 \%$ vs $82.8 \%$; $P=.02)$, were younger (median age: 67 years [IQR, $60-73$ years] vs 68 years [IQR, 61-75 years]; $P<.001$ ), and tended to have less delay to start of chemotherapy $(20.3 \%$ vs $23.4 \%$ of patients in quartile $4 ; P=.01$ ). Otherwise, the distribution of patient demographic characteristics was similar (Table 1).

\section{Survival Outcomes}

Median follow-up was 23.5 months (95\% CI, 22.524.6 months). Kaplan-Meier survival curves for the national cohort are displayed in Figure 2. Median OS was 12.1 months (95\% CI, 11.2-12.9 months) in the carboplatin/ pemetrexed/bevacizumab group compared with 8.6 months (95\% CI, 8.1-9.1 months) in the carboplatin/pemetrexed group. The unadjusted HR was 0.81 (95\% CI, 0.75-0.87). In multivariable Cox proportional hazards models adjusting for baseline characteristics, the adjusted $\mathrm{HR}$ was 0.80 (95\% CI, 0.75-0.86; Table 2). There was no statistically significant interaction between age and bevacizumab use.

\section{Sensitivity Analyses}

Although the absence of a recorded ECOG PS was not associated with receipt of bevacizumab, given the 


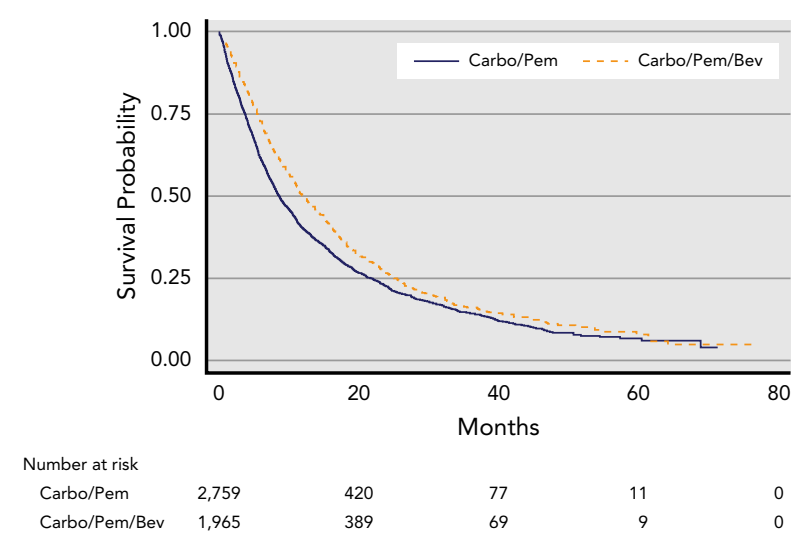

Figure 2. Kaplan-Meier survival curve for patients with metastatic nonsquamous non-small cell lung cancer in the national cohort by first-line chemotherapy administration \pm bevacizumab.

Abbreviations: Bev, bevacizumab; Carbo, carboplatin; Pem, pemetrexed.

substantial proportion of patients with missing PS, we performed a sensitivity analysis that included only patients with known PS $(n=2,390)$. After adjusting for the same characteristics listed in Table 2, the addition of bevacizumab to carboplatin/pemetrexed remained associated with improved OS with a similar effect size (HR, 0.80; 95\% CI, 0.72-0.88). We also performed a sensitivity analysis with extreme case imputation. When adjusting for the same demographic and clinical characteristics described earlier in a Cox proportional hazards model using the imputed values for ECOG PS, bevacizumab use remained beneficial, although with a higher HR $(0.85 ; 95 \%$ CI, $0.78-0.93$ ).

In addition to the sensitivity analyses, we conducted a secondary analysis of patients treated at the University of Pennsylvania and thus had detailed EHR data available for manual abstraction by the investigators $(n=539$; these patients were not included in the national cohort). Baseline characteristics are listed in Table 3. We used a Cox proportional hazards model that adjusted for the same characteristics listed in Table 1, but also for presence of brain metastases and hemoptysis and anticoagulation use. As expected, patients with these factors were less likely to receive bevacizumab (Table 3). Using the expanded model, the beneficial effect of bevacizumab on OS persisted (median OS, 18.0 vs 10.0 months; adjusted HR, 0.75; 95\% CI, 0.59-0.96; $P=.02)$.

\section{Subgroup Analyses}

Because of potential differences in the efficacy and risk profile of bevacizumab in the elderly, we performed subgroup analyses in the national cohort restricted to patients aged $\geq 65$ years $(n=2,966)$ and $\geq 75$ years $(\mathrm{n}=1,168)$. Kaplan-Meier curves are presented in Figure 3. In multivariable analysis, the $\mathrm{HR}$ associated with bevacizumab use was 0.80 (95\% CI, 0.73-0.88) in patients aged $\geq 65$ years and 0.77 (95\% CI, $0.67-0.89$ ) in those aged $\geq 75$ years.

\section{Discussion}

Although there has never been a RCT to demonstrate a survival benefit associated with the addition of bevacizumab to carboplatin/pemetrexed for advanced nonsquamous NSCLC, this triplet regimen is administered commonly in clinical practice. ${ }^{6}$ We evaluated the effectiveness of bevacizumab in combination with carboplatin/pemetrexed in a nationally representative cohort of patients with advanced nonsquamous NSCLC. The addition of bevacizumab was associated with improved OS, even after adjusting for known prognostic variables. Furthermore, in a secondary analysis of patients treated at a single academic institution, this survival benefit was maintained after adjusting for potential confounders of the relationship between bevacizumab use and survival, including brain metastases, anticoagulation use, and recent history of hemoptysis (although an excess of $2.5 \mathrm{~mL}$ within the past 3 months is traditionally considered a contraindication to bevacizumab, it is still sometimes offered to patients with scant, clinically insignificant amounts of hemoptysis). ${ }^{16}$ Bevacizumab was also beneficial in subgroups limited to elderly patients. In light of the increased toxicity and financial burden associated with bevacizumab, ${ }^{17-19}$ our study, despite the drawbacks of a retrospective analysis, helps address an important evidence gap in the management of advanced nonsquamous NSCLC.

Although an OS advantage was recently demonstrated from the addition of pembrolizumab to carboplatin/pemetrexed as first-line treatment in patients with metastatic nonsquamous NSCLC, regardless of tumor PD-L1 expression, ${ }^{8}$ bevacizumab may still have a role in patient treatment. Certain patients, such as those with severe/active autoimmune disease, interstitial lung disease, or prior organ transplant, are generally not candidates for immunotherapy. ${ }^{20}$ In addition, the role of immune checkpoint inhibitors in patients with poor PS remains unclear, because patients with an ECOG PS $\geq 2$ were generally excluded from immunotherapy trials. ${ }^{8}$ There are also parts of the world where bevacizumab is more readily available than pembrolizumab for economic or logistical reasons. Finally, it is possible that bevacizumab use may soon increase based on a recent randomized phase III trial demonstrating improved OS with the addition of the PD-L1 inhibitor atezolizumab to bevacizumab plus chemotherapy in patients 
Table 2. Crude Median OS and Adjusted HR for OS

Crude Median OS $(95 \% \mathrm{Cl})$, mo

\begin{tabular}{|c|c|c|c|}
\hline Characteristic & Carboplatin/Pemetrexed & Carboplatin/Pemetrexed/Bevacizumab & HR $(95 \% \mathrm{Cl})$ \\
\hline \multicolumn{4}{|l|}{ Chemotherapy regimen } \\
\hline Carboplatin/Pemetrexed & $8.6(8.1-9.1)$ & & Ref \\
\hline Carboplatin/Pemetrexed/Bevacizumab & & $12.1(11.2-12.9)$ & $0.80(0.75-0.86)$ \\
\hline \multicolumn{4}{|l|}{ Age, y } \\
\hline$<55$ & $8.2(7.1-11.2)$ & $11.3(9.2-14.3)$ & Ref \\
\hline $55-64$ & 8.5 (7.5-9.5) & $12.4(10.9-14.4)$ & $0.97(0.85-1.10)$ \\
\hline $65-74$ & $8.8(8.0-9.9)$ & $12.1(10.9-13.4)$ & $0.96(0.85-1.09)$ \\
\hline$\geq 75$ & $8.4(7.6-9.7)$ & $12.1(10.7-14.2)$ & $1.0(0.89-1.16)$ \\
\hline \multicolumn{4}{|l|}{ Sex } \\
\hline Female & $10.0(9.1-11.0)$ & $15.1(13.4-16.3)$ & Ref \\
\hline Male & $7.7(7.0-8.2)$ & $10.3(9.1-11.2)$ & $1.27(1.18-1.36)$ \\
\hline \multicolumn{4}{|l|}{ Race } \\
\hline White & $8.6(8.0-9.1)$ & $12.3(11.3-13.6)$ & Ref \\
\hline Black & $10.8(8.1-12.8)$ & $11.9(8.2-15.2)$ & $0.91(0.79-1.04)$ \\
\hline Asian & $11.9(7.0-31.9)$ & $17.1(9.2-26.6)$ & $0.70(0.51-0.96)$ \\
\hline Other & $9.5(7.5-12.6)$ & $11.3(9.9-13.3)$ & $1.06(0.93-1.20)$ \\
\hline Unknown & $7.7(6.7-8.7)$ & $11.0(9.0-13.1)$ & $1.06(0.97-1.17)$ \\
\hline \multicolumn{4}{|l|}{ Stage } \\
\hline Metastatic recurrence of stage I-III & $16.3(13.6-18.3)$ & $15.9(13.3-17.7)$ & Ref \\
\hline Initial stage IV & $7.7(7.2-8.2)$ & $11.3(10.9-12.4)$ & $1.57(1.43-1.74)$ \\
\hline \multicolumn{3}{|c|}{ Days from metastatic diagnosis to chemotherapy initiation } & $0.92(0.88-0.97)$ \\
\hline Quartile 1 (0-21) & $7.2(6.6-8.1)$ & $11.3(10.3-13.0)$ & Ref \\
\hline Quartile 2 (22-33) & $9.2(8.2-11.2)$ & $12.9(11.2-14.8)$ & $0.88(0.80-0.97)$ \\
\hline Quartile $3(34-50)$ & $8.3(7.5-9.2)$ & $12.1(11.0-13.8)$ & $0.96(0.87-1.05)$ \\
\hline Quartile 4 (51-120) & $10.0(8.7-11.3)$ & $11.5(10.0-14.3)$ & $0.84(0.76-0.93)$ \\
\hline \multicolumn{4}{|l|}{ ECOG performance status } \\
\hline 0 & $14.2(11.5-16.5)$ & $15.9(13.0-19.3)$ & Ref \\
\hline 1 & $8.4(7.6-9.4)$ & $11.8(10.8-13.7)$ & $1.41(1.26-1.57)$ \\
\hline 2 & $5.8(4.7-6.8)$ & $6.7(5.6-9.1)$ & $1.96(1.70-2.26)$ \\
\hline $3-4$ & $1.4(1.1-4.2)$ & $3.3(2.0-6.2)$ & $3.65(2.84-4.72)$ \\
\hline Missing & $8.3(7.7-9.0)$ & $11.7(10.9-13.2)$ & $1.43(1.30-1.58)$ \\
\hline
\end{tabular}

Abbreviations: HR, hazard ratio; OS, overall survival.

with metastatic nonsquamous NSCLC. ${ }^{21}$ However, caution should be taken in extrapolating the results of our study to patients receiving immunotherapy, because we excluded patients receiving PD-1/PD-L1 inhibitors.

Strengths of our study include use of a contemporary and nationally representative cohort, which speaks to the generalizability of our findings, and the large sample size, which afforded the power to detect a small OS benefit. The primary limitation of our study, based on its retrospective and nonrandomized nature, is the potential for unmeasured confounding. Although we adjusted for ECOG PS, which may address this issue to some extent, PS data were missing for approximately half of the patients. This is a direct result of EHR-derived data, because PS is only available if recorded by the patient's providers. We attempted to address this limitation using sensitivity analyses, in which the beneficial effect associated with bevacizumab was maintained. In addition, the survival benefit associated with bevacizumab persisted in the University of Pennsylvania cohort after adjusting for brain metastases, hemoptysis, and anticoagulation use, which are 3 important potential confounders in this analysis. ${ }^{22}$ 


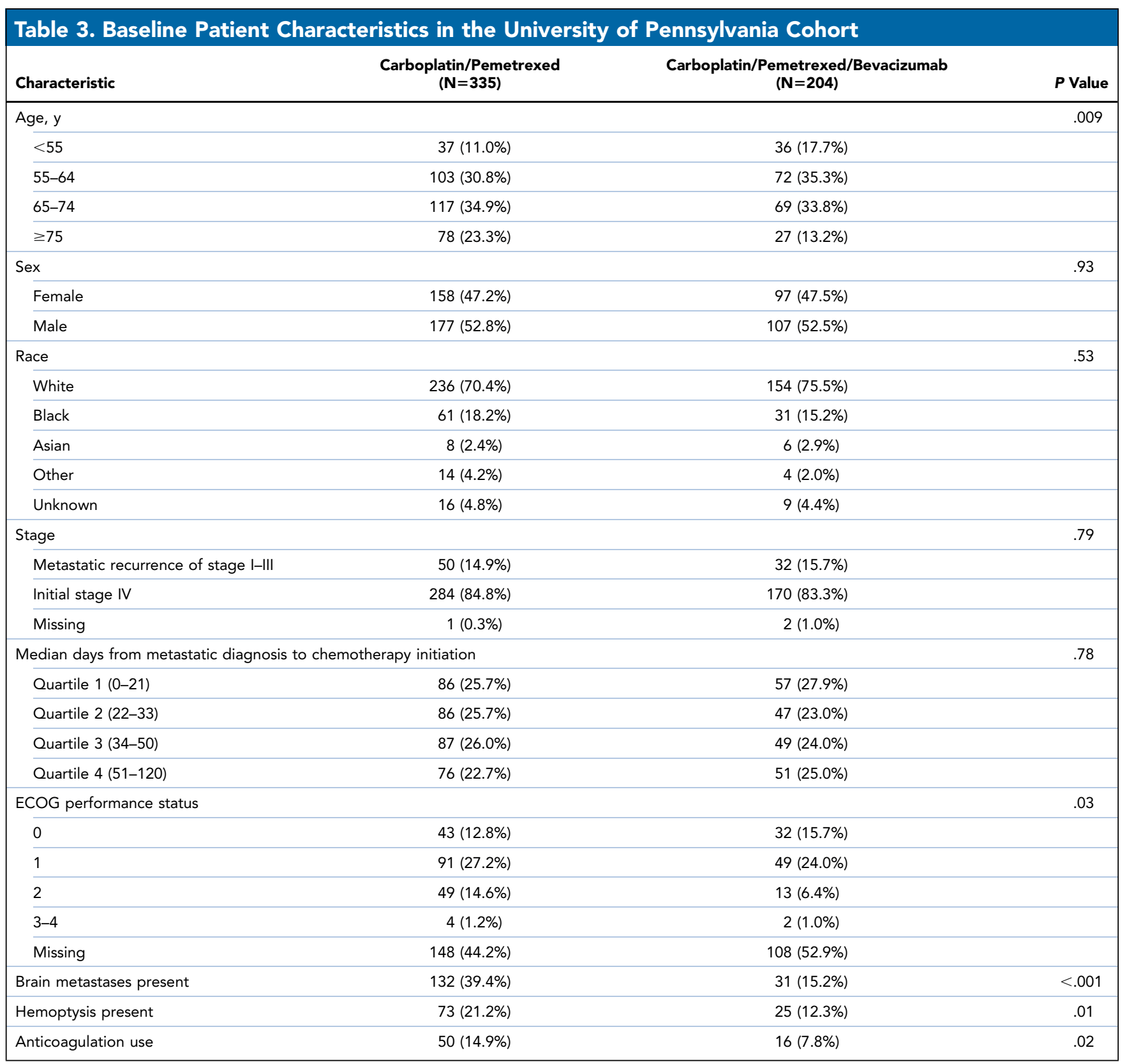

Nonetheless, we cannot rule out the possibility that unmeasured confounders, particularly other medical comorbidities not available in the national cohort data, are responsible for the association between bevacizumab use and improved OS. In addition, the University of Pennsylvania cohort was younger in general compared with the national cohort, which may have mitigated the impact of brain metastases, hemoptysis, and anticoagulation use on outcomes in the institutional analysis.

Another limitation is that we did not seek to assess the impact of the number of chemotherapy cycles received, the duration of bevacizumab treatment, or the use of maintenance therapy, all of which could impact outcomes in this population. ${ }^{23}$ However, this was an intention-to-treat analysis, and the primary aim was to determine the real-world effectiveness of the addition of bevacizumab to carboplatin/ pemetrexed. It is debatable whether the median OS advantage of 3.5 months (HR, 0.80) associated with bevacizumab in our study is clinically meaningful. Although this degree of incremental benefit is considered "clinically meaningful" according to prior ASCO guidance, ${ }^{24}$ and exceeds the median survival 
A

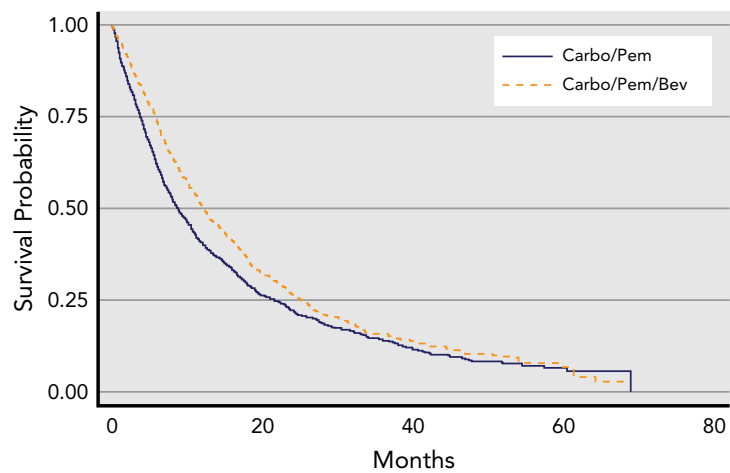

B

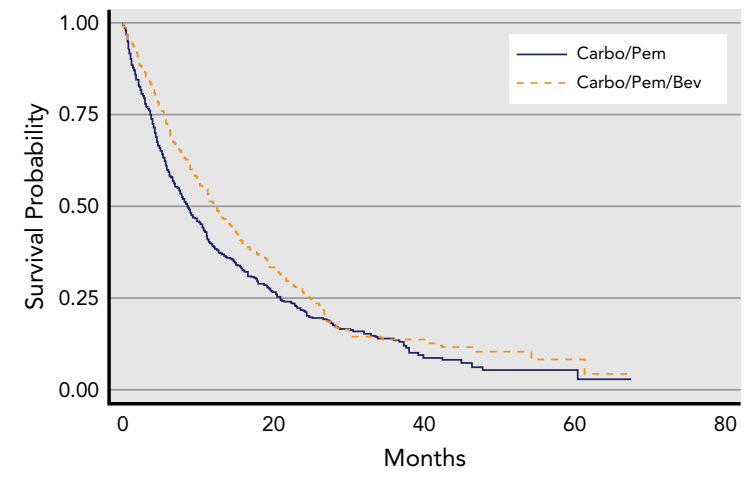

Number at risk

$\begin{array}{lrrrrr}\text { Carbo/Pem } & 767 & 101 & 14 & 3 & 0\end{array}$
Number at risk

Carbo/Pem 1,791

Carbo/Pem/Bev 1,175
270

53

40
7

Figure 3. Kaplan-Meier survival curves for patients aged $(A) \geq 65$ years and $(B) \geq 75$ years with metastatic nonsquamous non-small cell lung cancer in the national cohort by first-line chemotherapy administration \pm bevacizumab.

Abbreviations: Bev, bevacizumab; Carbo, carboplatin; Pem, pemetrexed.

increment observed in ECOG 4599, ${ }^{11}$ the financial cost and increased toxicity associated with bevacizumab must also be considered when deciding whether to use bevacizumab both in individual patients and for society as a whole. ${ }^{25}$

\section{Conclusions}

This is the first adequately powered study to address whether the addition of bevacizumab to carboplatin/ pemetrexed is associated with OS in advanced nonsquamous NSCLC. It is unlikely that an RCT to address this question will ever be performed due to the size and cost that such a trial would entail, and the higher priority of other clinical questions in thoracic oncology. Results of our real-world study provide essential information that can be incorporated into the complex clinical decision-making necessary for this large population of patients with cancer.

Submitted August 29, 2018; accepted for publication October 25, 2018.

Author contributions: Study concept and design: Bagley, Cohen, Langer. Data acquisition: Talento, Meropol. Data analysis and interpretation: Bagley, Mitra, Vachani. Manuscript preparation: Bagley. Critical revision: All authors.

Disclosures: Dr. Bagley has disclosed that he has received grant/research support from Eli Lilly. Dr. Meropol has disclosed that he is an employee of and has stock options in Flatiron Health, Inc., an independent subsidiary of the Roche Group. Dr. Langer has disclosed that he has received grant/research support from and served as a scientific advisor for Roche/Genentech and Eli Lilly. The remaining authors have not received any financial consideration from any person or organization to support the preparation, analysis, results, or discussion of this article.

Funding: This work was supported by a grant from the $\mathrm{NCl}$ (T32 CA009679) and the National Institute of Environmental Health Sciences (P30-ES013508).

Correspondence: Stephen J. Bagley, MD, MSCE, Division of Hematology/ Oncology, Perelman School of Medicine at the University of Pennsylvania, 3400 Civic Center Boulevard, Philadelphia, PA 19104. Email: stephen.bagley@uphs.upenn.edu

\section{References}

1. Herbst RS, Morgensztern D, Boshoff C. The biology and management of non-small cell lung cancer. Nature 2018;553:446-454.

2. Hanna N, Johnson D, Temin S, et al. Systemic therapy for stage IV nonsmall-cell lung cancer: American Society of Clinical Oncology clinical practice guideline update. J Clin Oncol 2017;35:3484-3515.

3. Ettinger DS, Wood DE, Aisner DL, et al. NCCN Clinical Practice Guidelines in Oncology: Non-Small Cell Lung Cancer. Version 1.2019. Accessed October 23, 2018. To view the most recent version of these guidelines, visit NCCN.org.

4. Scagliotti GV, Parikh P, von Pawel J, et al. Phase III study comparing cisplatin plus gemcitabine with cisplatin plus pemetrexed in chemotherapy-naive patients with advanced-stage non-small-cell lung cancer. J Clin Oncol 2008;26:3543-3551.

5. Gyawali B, Prasad V. Pemetrexed in nonsquamous non-small-cell lung cancer: the billion dollar subgroup analysis. JAMA Oncol 2018;4:17-18.

6. Abernethy AP, Arunachalam A, Burke $T$, et al. Real-world first-line treatment and overall survival in non-small cell lung cancer without known EGFR mutations or ALK rearrangements in US community oncology setting. PLoS One 2017;12:e0178420.

7. Barlesi F, Scherpereel A, Rittmeyer A, et al. Randomized phase III trial of maintenance bevacizumab with or without pemetrexed after first-line induction with bevacizumab, cisplatin, and pemetrexed in advanced nonsquamous non-small-cell lung cancer: AVAPERL (MO22089). J Clin Oncol 2013;31:3004-3011.

8. Gandhi L, Rodríguez-Abreu D, Gadgeel S, et al. Pembrolizumab plus chemotherapy in metastatic non-small-cell lung cancer. N Engl J Med 2018;378:2078-2092.

9. Abdel-Wahab N, Shah M, Lopez-Olivo MA, et al. Use of immune checkpoint inhibitors in the treatment of patients with cancer and preexisting autoimmune disease: a systematic review. Ann Intern Med 2018, 168:121-130.

10. Kurtovic-Kozaric A, Vranic S, Kurtovic S, et al. Lack of access to targeted cancer treatment modalities in the developing world in the era of precision medicine: real-life lessons from Bosnia. J Glob Oncol 2018;4:1-5.

11. Sandler A, Gray R, Perry MC, et al. Paclitaxel-carboplatin alone or with bevacizumab for non-small-cell lung cancer. N Engl J Med 2006;355: 2542-2550. 
12. Patel JD, Socinski MA, Garon EB, et al. PointBreak: a randomized phase III study of pemetrexed plus carboplatin and bevacizumab followed by maintenance pemetrexed and bevacizumab versus paclitaxel plus carboplatin and bevacizumab followed by maintenance bevacizumab in patients with stage IIIB or IV nonsquamous non-small-cell lung cancer. J Clin Oncol 2013;31:4349-4357.

13. Flatiron Health. Available at: https://flatiron.com/real-world-evidence/. Accessed October 23, 2018.

14. Berger ML, Curtis MD, Smith G, et al. Opportunities and challenges in leveraging electronic health record data in oncology. Future Oncol 2016; 12:1261-1274.

15. Curtis MD, Griffith SD, Tucker M, et al. Development and validation of a high-quality composite real-world mortality endpoint. Health Serv Res 2018;53:4460-4476.

16. Reck M, Barlesi F, Crinò L, et al. Predicting and managing the risk of pulmonary haemorrhage in patients with NSCLC treated with bevacizumab: a consensus report from a panel of experts. Ann Oncol 2012;23:1111-1120.

17. Spigel DR, Hainsworth JD, Joseph MJ, et al. Randomized phase 2 trial of pemetrexed, pemetrexed/bevacizumab, and pemetrexed/carboplatin/ bevacizumab in patients with stage IIIB/IV non-small cell lung cancer and an Eastern Cooperative Oncology Group performance status of 2. Cancer 2018;124:1982-1991.

18. Gautschi O, Rothschild SI, Li Q, et al. Bevacizumab plus pemetrexed versus pemetrexed alone as maintenance therapy for patients with advanced nonsquamous non-small-cell lung cancer: update from the Swiss Group for Clinical Cancer Research (SAKK) 19/09 trial. Clin Lung Cancer 2017;18:303-309.

19. Goulart B, Ramsey S. A trial-based assessment of the cost-utility of bevacizumab and chemotherapy versus chemotherapy alone for advanced non-small cell lung cancer. Value Health 2011;14:836-845.

20. Johnson DB, Sullivan RJ, Menzies AM. Immune checkpoint inhibitors in challenging populations. Cancer 2017;123:1904-1911.

21. Socinski MA, Jotte RM, Cappuzzo F, et al. Atezolizumab for first-line treatment of metastatic nonsquamous NSCLC. N Engl J Med 2018;378: 2288-2301.

22. Kurzrock R, Stewart DJ. Exploring the benefit/risk associated with antiangiogenic agents for the treatment of non-small cell lung cancer patients. Clin Cancer Res 2017;23:1137-1148.

23. Stinchcombe TE, Socinski MA. Maintenance therapy in advanced nonsmall cell lung cancer: current status and future implications. J Thorac Oncol 2011;6:174-182

24. Ellis LM, Bernstein DS, Voest EE, et al. American Society of Clinical Oncology perspective: raising the bar for clinical trials by defining clinically meaningful outcomes. J Clin Oncol 2014;32:1277-1280.

25. Gilden DM, Kubisiak JM, Pohl GM, et al. Treatment patterns and costeffectiveness of first line treatment of advanced non-squamous nonsmall cell lung cancer in Medicare patients. J Med Econ 2017;20: $151-161$ 